\title{
Dual Inhibition of Anti-apoptotic Proteins BCL-XL and MCL-1 Enhances Cytotoxicity of Nasopharyngeal Carcinoma Cells
}

\section{Siti Fairus Abdul Rahman}

Universiti Sains Malaysia

\section{Azali Azlan}

Universiti Sains Malaysia

\section{Kwok-Wai Lo}

Chinese University of Hong Kong

\section{Ghows Azzam}

Universiti Sains Malaysia

Nethia Mohana-Kumaran ( $\nabla$ nethiakumaran@usm.my)

Universiti Sains Malaysia

\section{Short Report}

Keywords: Nasopharyngeal carcinoma, BCL-XL, MCL-1, BFL-1, BH3 mimetics, spheroids

Posted Date: November 18th, 2021

DOI: https://doi.org/10.21203/rs.3.rs-1085342/v1

License: (9) This work is licensed under a Creative Commons Attribution 4.0 International License. Read Full License 


\section{Abstract}

One of the many strategies that cancer cells evade death is through up-regulation of the BCL-2 antiapoptotic proteins. Hence, these proteins have become attractive therapeutic targets. Given that different cell population rely on different anti-apoptotic proteins for survival, it is crucial to determine which proteins are important for Nasopharyngeal carcinoma (NPC) cell survival. Here we determined the survival requirements for the NPC cells using combination of the CRISPR/Cas9 technique and selective BH3-mimetics. A human apoptosis $\mathrm{RT}^{2}$ Profiler PCR Array was first employed to profile the anti-apoptotic gene expressions in NPC cell lines HK-1 and C666-1. The HK-1 cells expressed all the anti-apoptotic genes (MCL-1, BFL-1, BCL-2, BCL-XL, and BCL-w). Similarly, the C666-1 cells expressed all the anti-apoptotic genes except $B F L-1$ (undetectable level). Notably, both cell lines highly expressed $M C L-1$. Deletion of MCL-1 sensitized the NPC cells to BCL-XL selective inhibitor A-1331852, suggesting that MCL-1 and BCLXL may be important for NPC cell survival. Co-inhibition of MCL-1 and BCL-2 with MCL-1 selective inhibitor 563845 and BCL-2 selective inhibitor ABT-199 inhibited NPC cell proliferation but the effect on cell viability was more profound with co-inhibition of MCL-1 and BCL-XL with S63845 and A-1331852, implying that MCL-1 and BCL-XL are crucial for NPC cell survival. Furthermore, co-inhibition of MCL-1 and BCL-XL inhibited the growth and invasion of NPC spheroids. Deletion of BFL-1 sensitized NPC cells to A1331852 suggesting that BFL-1 may play a role in NPC cell survival. Taken together co-inhibition of BCL$\mathrm{XL}$ and MCL-1/BFL-1 could be potential treatment strategies for NPC.

\section{Introduction}

NPC is a rare cancer worldwide but endemic in Asia especially in South-East Asia [1]. Concurrent chemoradiotherapy (CCRT) plus adjuvant chemotherapy has been the standard of care treatment for NPC since it was established by the intergroup 0099 [2]. However, treating patients with metastatic NPC is often a challenge as patients tend to develop resistance to cisplatin and radiotherapy. Hence, improved treatment strategies are needed for better patient survival.

The BCL-2 family of proteins are critical regulators of the apoptosis pathway. The members of the family are grouped into pro- and anti-apoptotic proteins [3]. The anti-apoptotic proteins (BCL-2, BCLXL, BCL-W, $M C L-1$ and BFL-1) are up-regulated in many cancers and hence have emerged as potential therapeutic targets. Initial studies on the expressions of the BCL-2 family proteins in NPC, focused on BCL-2. Immunohistochemistry (IHC) technique was unanimously employed to determine the expression level of BCL-2 in NPC tissues. Using IHC, BCL-2 expression was detected in $80 \%$ NPC tissues and $71 \%$ adjacent dysplastic lesions compared to only $37 \%$ in nasopharynx epithelia (NPE) and $30 \%$ in NPE of chronically inflamed nasopharynx patients [4]. In a separate study 31 of 51 NPC tumour samples tested, expressed BCL-2 [5]. High expression of BCL-2 was also observed in a large pool of NPC tissues of 148 samples compared to 164 samples of non-cancerous NPC tissues [6]. Patients with negative BCL-2 expression were reported to display better disease free 5-year survival compared to patients with BCL-2 positive tumours [7]. 
There were number of studies which attempted to target the anti-apoptotic proteins for NPC treatment but the study findings were unsatisfactory [8-10]. The problem lied largely on the failure to determine which anti-apoptotic proteins did NPC cells relied for survival. A recent finding demonstrated that co-inhibition of MCL-1 and BCL-2 killed NPC cells in both in vitro and in vivo models [11]. However, the role of BCL-XL for NPC cell survival was not interrogated in the same study. Our own study unveiled that co-inhibition of BCL-2 and BCL-XL did not induce cell killing in NPC [10]. This led to the re-evaluation of the utility of BCL2 and BCL-XL as therapeutic targets in NPC. Lian et al, study findings raised two questions (1) do NPC cells depend on other anti-apoptotic proteins for survival? (e.g. MCL-1 or BFL-1); OR (2) do they depend on combination of two anti-apoptotic proteins that are not partly targeted, by ABT-263, for example MCL-1 and BCL-XL? This study primarily focused on investigating the functional relevance of $M C L-1$ and BFL-1, given the limited efforts in addressing their roles in NPC survival.

There are a few strategies to determine the dependencies of cancer cells to anti-apoptotic protein(s) namely the dynamic $\mathrm{BH} 3$ profiling technique [12], the CRISPR/Cas9 genome editing technique [13] and chemical parsing approach using $\mathrm{BH} 3$-mimetics, given their selectivity in inhibiting specific anti-apoptotic proteins [14]. This study utilized combination of the CRISPR/Cas9 technique and BH3-mimetics to delineate the contributions of the anti-apoptotic proteins for NPC cell survival. The functional relevance of MCL-1 and BFL-1 for NPC cell survival were first determined using the CRISPR/Cas9 technique and later the parental and $M C L-1 / B F L-1$ manipulated NPC cells were subjected to treatment with selective BH3mimetics to complement the gene editing studies and to also investigate the translational relevance of the BH3-mimetics for NPC management.

\section{Material And Methods}

\subsection{Cell culture}

The HK-1 and C666-1 cell lines were cultured in RPMI 1640 medium (supplemented with $10 \%$ heated foetal bovine serum (FBS). Additionally, 10\% (v/v) Glutamax was added to the RPMI complete medium that was used to grow the C666-1 cells. NPC cell lines HK-1 and C666-1 were authenticated using the AmpFISTR profiling [15]. All cell culture consumables were purchased from Thermo Fisher Scientific, MA, USA.

\subsection{Custom $\mathrm{RT}^{2}$ Profiler PCR Array}

Total RNA from the HK-1 and C666-1 parental cell lines were extracted with TRIzol reagent (Invitrogen). RNA purity was quantified using the NanoDrop spectrophotometer (Thermo Fischer Scientific) and the PCR array (customized human apoptosis profiler array designed by Qiagen, Hilden, Germany) was conducted as per the manufacturer's protocol. Data was analysed using web-based analysis tools.

\section{3 sgRNA design and cloning}


The CHOP CHOP design software was employed to design sgRNAs (https://chopchop.cbu.uib.no/). Two sgRNAs were designed for each gene of interest. The sgRNAs were cloned into expression vector plasmid, pSpCas9(BB)-2A-Puro (Px459) (Addgene). The sgRNAs oligo design included a 4-bp overhang for the forward (CACC) and complementary reverse (CAAA) to allow cloning at the BbSl-site of the vector plasmid. The sgRNAs sequences for MCL-1 sgRNA1: 5'-CACCCTTATAGGTATCCACATCCG-3'; MCL-1 sgRNA2: 5' CACCGTCCTACAGATACCACAACC-3'. The sgRNAs sequences for BFL-1 sgRNA1: 5'CACCGGGAGGGCGACTTTTGGCTA-3'; BFL-1 sgRNA2: 5'- CACCGGAGCTGGACGGGTACGAGC - 3'. The sgRNA sequences were verified with U6 primers (forward primer: GAGGGCTATTTCCATGATTCC, reverse primer: GCAACACACAACATCTCCA) for successful cloning.

\subsection{Cell transfection}

The MCL-1 sgRNAs or the BFL-1 sgRNAs were introduced into NPC HK-1 cells via transfection using Lipofectamine 2000 (Thermo Fisher Scientific). The HK-1 cells were plated at $1 \times 10^{5}$ cells per well in 24well plates. Three micrograms of DNA of vector carrying either the MCL-1 or BFL-1 sgRNAs with $3 \mu \mathrm{l}$ Lipofectamine 2000 were transfected into each well; cells were left for 72 hours. After 72 hours, transfected cells were selected with $1 \mathrm{ml}$ of serum reduced media supplemented with $1 \mathrm{ug} / \mathrm{ml}$ Puromycin (Thermo Fisher Scientific). The selection lasted for 48 hours. Post selection, complete media was added into each well. Once each well reached about $80 \%$ confluency, DNA and RNA were extracted from the manipulated cells to validate the gene knockouts.

\subsection{Validation of the MCL-1 and BFL-1 knockouts}

MCL-1 and BFL-1 mutagenesis were verified by conventional Sanger DNA sequencing and quantitative PCR (qPCR). The genomic knockouts of both genes were screened with customized PCR primers approximately 100-200 bp within the CRISPR cut site. MCL-1 sgRNA1: Forward oligo: 5' - GTT TGG CCT CAA AAG AAA CG - 3'; Reverse oligo: 5' - CTC TCT ATC CCC CTC CCC - 3'. MCL-1 sgRNA2: Forward oligo: 5' - CCG CTT GAG GAG ATG GAA G - 3'; Reverse oligo: 5' - TAT TGT GGT CAT GCC TGC CCG - 3'. BFL-1 sgRNA1: Forward oligo: 5' - AGC CTC CGT TTT GCC TTA TC- 3'; Reverse oligo: 5' - GAA GGG GTC AAT TAC TAC GG - 3'. BFL-1 sgRNA2: Forward oligo: 5' - TCT CAG CAC ATT GCC TCA AC - 3'; Reverse oligo: 5' - TCG TTT TGC AGG TCT CAC GA - 3'. During the PCR assay, Taq polymerase (Intron Biotechnology) was used to generate the 3 'A overhangs for TA cloning. The PCR products were purified and ligated into the PGEM-T Easy Vector (Promega) and later transformed into the Rubidium Chloride competent cells. The transformation culture was plated onto LBampicillin/IPTG/X-Gal plates.

Successfully cloned colonies appeared white. Colonies with correct insert size were sent for sequencing (First Base). The mutated DNAs of the colonies were compared to the parental sequences to affirm the knockouts and check for presence of InDels. Parallel to gene sequencing, gene expression quantification was performed via qPCR. The qPCR primers were customized considering the sgRNA oligo cute sites for MCL-1 and BFL-1. GAPDH was used as the housekeeping gene and the parental cells served as controls. Primers sequences are as follows: MCL-1.Forward oligo: 5' - GCG GTA ATC GGA CTC AAC CT - 3'; Reverse oligo: 5' - GTA GCC AAA AGT CGC CCT CC - 3'. BFL-1: Forward oligo: 5' - GCT GGC TCA GGA 
CTA TCT GC - 3'; Reverse oligo: 5' - TGG ACG TTT TGC TTG GAC CT - 3'. GAPDH: Forward oligo: 5' GTC TCC TCT GAC TTC AAC AGC G - 3'; Reverse oligo: 5' - ACC ACC CTG TTG CTG TAG CCA A - 3’.

\subsection{Cytotoxicity Assay}

Manipulated and parental cells were either treated with BCL-2 selective inhibitor ABT-199, BCL-XL inhibitor A-1331852 and MCL-1 selective inhibitor S63845, alone and in combinations. All drugs were purchased from MedChem Express, NJ, USA. Drug sensitivity assays were conducted as described previously [16]. Average $\mathrm{IC}_{50}$ values were calculated from the experimental data. In the drug response curves shown, the $y$ axis represents cell proliferation, with cell proliferation of the untreated controls representing $100 \%$. The $x-$ axis was formatted to have a base 10 logarithmic scale but the drug concentrations shown were not logtransformed prior to plotting of the graphs. Synergistic interaction between the drug combination were analysed using the CalcuSyn software (Version 2.11, Biosoft Inc, Cambridge, UK).

\subsection{Three-dimensional spheroids}

Spheroids were generated as described previously [17]. Live-dead staining were conducted as described previously [18]. Spheroids were treated with A-1331852 and S63845, alone and in combination for $72 \mathrm{~h}$ and over 10 days. Spheroid images were taken using the Nikon-300 inverted fluorescence microscope. For quantification of green and red signals, an outline was drawn around each spheroid in a focal Z plane which showed the maximum size. Area and mean green/red fluorescence was measured, along with adjacent background readings for control spheroids, spheroids treated with either S63845 or A-1331852 and spheroids treated with combination of the two drugs. The total corrected green/red fluorescence $(T C R F)=$ integrated density - (area of selected cell $\times$ mean fluorescence of background readings) [19].

\section{Results}

\subsection{Expression of the BCL-2 anti-apoptotic proteins in the NPC cell lines}

The basal expressions of all the anti-apoptotic genes in the NPC cell lines HK-1 and C666-1 were first determined using a human apoptosis real time PCR array. In the HK-1 cells all the anti-apoptotic genes were detected. Most of the anti-apoptotic genes required more than 20 cycles of PCR amplification to detect except for MCL-1 which was detectable within 18 cycles of PCR amplification, which indicated high expression level of this gene in the cells (Fig. 1a). In the C666-1 cells, all of the anti-apoptotic genes

were detectable except BFL-1. Similar to the HK-1 cells, MCL-1 was detected within 20 cycles in the C6661 cells (Fig. 1b). Taken together, data shows that both NPC cell lines expressed all of the anti-apoptotic genes, except for BFL-1 in the C666-1 cells. More notably, both NPC cell lines expressed high levels of $M C L-1$. At the protein level, both cell lines expressed the anti-apoptotic protein MCL-1. High expression level of BCL-2 was detected in the HK-1 cells, whereas relatively high expression level of BCL-XL was detected in the C666-1 cells (Fig. 1c).

\subsection{Inhibition of either MCL-1, BCL-2 or BCL-XL alone is not sufficient to kill NPC cells}


Given the high expression levels of $M C L-1$ in both NPC cell lines, the functional relevance of $M C L-1$ for NPC cell survival was determined by deleting the gene in the NPC cell lines, using the CRISPR/Cas 9 technique. The NPC cell lines HK-1 and C666-1 were transfected with two independent single-guide RNAs (sgRNAs) targeting different regions of the human $M C L-1$ gene (hereafter the sgRNAs will be referred to as sgMCL-1\#1 and sgMCL-1\#2). MCL-1 mutagenesis was verified by conventional Sanger DNA sequencing and gene expression of $M C L-1$ in the parental and MCL-1 deleted cells were verified by qPCR. The HK-1 sgMCL-1\#2 cells demonstrated higher number of InDels compared to the HK-1 sgMCL-1\#1 (Fig. S1). The HK-1 sgMCL-1\#2 cells demonstrated complete reduction of MCL-1 compared to HK-1 sgMCL$1 \# 1$ cells which only resulted in $90 \%$ reduction in MCL-1 expression (Fig. 1d). The HK-1 sgMCL-1\#2 cells were viable despite complete reduction of MCL-1 indicating that the cells did not rely on MCL-1 alone for survival. The C666-1 sgMCL-1\#2 cells demonstrated higher number of InDels compared to the C666-1 sgMCL-1\#1 cells (Fig. S2a). The C666-1 sgMCL-1\#1 and sgMCL-1\#2 cells only resulted in $77 \%$ and $80 \%$, reduction in MCL-1 expression, respectively (Fig. S2b).

Given that the sgMCL-1\#2 demonstrated more profound reduction of $M C L-1$ expression, the HK-1/C666-1 sgMCL-1\#2 cells were treated with increasing concentrations of either ABT-199 or A-1331852 to access whether MCL-1 deletion sensitized NPC cells to these inhibitors. The HK-1 parental cells were resistant to single agent treatment of ABT-199 and A-1331852 (Fig. 1e). The HK-1 sgMCL-1\#2 cells were weakly sensitized to ABT-199 (Fig. 1e - red circle \& Table S1). However, the HK-1 sgMCL-1\#2 cells were sensitized to A-1331852 by 4-fold (Fig. 1e - purple circle \& Table S1), indicating that the MCL-1 and BCL-XL may be important for NPC cell survival. The C666-1 sgMCL-1\#2 cells were modestly sensitized to ABT-199 and A-1331852 (Fig. S2c), most probably due to incomplete reduction of MCL-1.

Taken together the gene editing findings were inconsistent. Only the HK-1 sg MCL-1\#2 cells were sensitized to A-1331852. Hence, there was a possibility that this could be a cell-type dependant effect. Given the incomplete reduction of MCL-1 in the C666-1 cells, it was challenging to delineate the role of MCL-1 and other anti-apoptotic proteins for NPC survival. Hence, to complement the gene editing study, the individual contribution of MCL-1, BCL-2 and BCL-XL for NPC cell survival was parsed using $\mathrm{BH} 3$ mimetics which selectively inhibit these proteins. The NPC cell lines HK-1 and C666-1 were first treated with single agent ABT-199, A-1331852 and S63845. The HK-1 (Fig. 1f) and C666-1 (Fig. 1g) cells were resistant to single agent treatment of all three $\mathrm{BH} 3$-mimetics suggesting that the cells depend on more than one anti-apoptotic protein for survival.

\subsection{NPC cell lines were sensitive to co-inhibition of MCL-1 and BCL-2}

Given that the NPC cells were insensitive to single agent treatment of the $\mathrm{BH} 3$ mimetics, the cells were first tested combination of ABT-199 and S63845. The HK-1 cells were treated with increasing concentrations of ABT-199 (0-32 $\mu \mathrm{M})$ in the absence and presence of either $0.5,1$ or $2 \mu \mathrm{M}$ of S63845 for 72 hours. At $0.5 \mu \mathrm{M}$ S63845, the cells were only sensitized to ABT-199 by 3-fold (Fig. 2a - open circle \& S. Table 2). The sensitization increased to 16 -fold at a concentration of $1 \mu \mathrm{M}$ (Fig. $2 \mathrm{a}-$ open triangle \& $\mathrm{S}$. Table 2) and $2 \mu \mathrm{M}$ of S63845 (Fig. 2a - open diamond \& S. Table 2). Drug interaction analyses were 
conducted using the CalcuSyn software (Version 2.11, Biosoft Inc, Cambridge, UK) to generate Fa-Cl isobologram plots. Points with $\mathrm{Cl}<1$ indicates synergistic drug interaction. Drug interaction analyses indicated that the drug combinations demonstrated synergism at multiple doses of combinations of ABT199 and S63845 in the HK-1 cells (Fig. 2b-d).

Similarly, the C666-1 cells were treated with increasing concentrations of ABT-199 (0-32 $\mu \mathrm{M})$ in the absence and presence of either $0.5,1$ or $2 \mu \mathrm{M}$ of $\mathrm{S} 63845$ for 72 hours. At $0.5 \mu \mathrm{M} \mathrm{S63845}$, the cells were sensitized to ABT-199 by 5-fold (Fig. 2e - open circle \& S. Table 2). The sensitization increased to 12 -fold at $1 \mu \mathrm{M}$ (Fig. $2 \mathrm{e}-$ open triangle \& S. Table 2) and increased further to 36 -fold at $2 \mu \mathrm{M}$ of S63845 (Fig. 2e - open diamond \& S. Table 2). Drug interaction analyses indicated that the drug combinations demonstrated synergism at multiple doses of combinations of ABT-199 and S63845 in the C666-1 cells (Fig 2f-h).

\subsection{Substantial inhibition of NPC cell proliferation driven by co-inhibition of MCL-1 and BCL-XL}

Next, the NPC cell lines were treated with increasing doses of A-1331852 (0-32 $\mu \mathrm{M})$ and fixed doses of S63845 $(0.5 \mu \mathrm{M}, 1 \mu \mathrm{M}$ or $2 \mu \mathrm{M})$ for 72 hours. In the HK-1 cells, the presence of $0.5 \mu \mathrm{M}$ S63845, complete $100 \%$ cell killing was achieved resulting in $0 \%$ cell viability (Fig. $2 \mathrm{i}$ - open square) and $\mathrm{S} 63845$ sensitized the cells to A-1331852 by $>15$-fold (Table S3). Similar data were obtained when the concentration of S63845 was increased to $1 \mu \mathrm{M}$ (Fig. $2 \mathrm{i}$ - open diamond \& Table S3) and $2 \mu \mathrm{M}$ (Fig. $2 \mathrm{i}$ - open triangle \& Table S3).

Similarly, in the C666-1 cells, combination with $\mathrm{S} 63845$ sensitized the cells to A-1331852 to all three concentrations tested. In the presence of $0.5 \mu \mathrm{M}$ S63845, there was complete loss of the dose-dependent curve resulting in $0 \%$ cell viability (Fig. $2 \mathrm{j}$ - open circle) and $\mathrm{S} 63845$ sensitized the cells to A-1331852 by $>29$-fold (Table S3). Similar data were obtained when the concentration of S63845 was increased to $1 \mu \mathrm{M}$ (Fig. $2 \mathrm{j}$ - open diamond \& Table S3) and $2 \mu \mathrm{M}$ (Fig. 2j - open triangle \& Table S3).

\section{S63845 sensitized spheroids to A-1331852 and vice versa}

Next, combination of S63845 and A-1331852 was tested on spheroids generated from the HK-1 cells. The HK-1 spheroids were treated with $\mathrm{S} 63845$ and A-1331852 alone and in combination, first, over 3 days and later, over 10 days, to observe for emergence of resistance cells.

Spheroids were insensitive to single agent treatment of S63845 and A-1331852 except at $2 \mu \mathrm{M}$ concentration of each drug (Fig. 3a). There was obvious sensitization of the spheroids to A-1331852 by S63845 at concentration of S63845 as low as $0.5 \mu \mathrm{M}$. Similarly, at $0.5 \mu \mathrm{M}$ of A-1331852, the spheroids

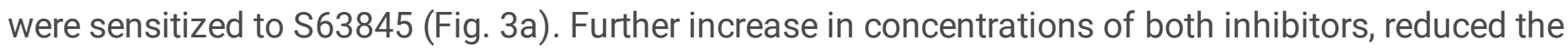
viability of the spheroids in a dose-dependent manner (Fig. 3a). This was evident with the decrease in green fluorescence intensity (Fig. 3b) but an increase in red fluorescence intensity (Fig. 3c), indicating the presence of more dead cells as the combination concentrations increased. Over 10 days, treatment with combination of S63845 and A-1331852 resulted in reduction of green fluorescence intensity and an 
increase in red fluorescence intensity (Fig. 4). Moreover, there were no emergence of viable cells in the highest combination concentration of both drugs, indicating there were no rapid generation of resistance cells over 10 days (Fig. 4).

Collectively, our data demonstrated that there was a greater response of the NPC cell lines to co-inhibition of MCL-1 and BCL-XL compared to co-inhibition of MCL-1 and BCL-2, suggesting that co-inhibition of MCL-1 and BCL-XL are better therapeutic targets for killing NPC cells.

\subsection{BFL-1 deleted NPC cells sensitized to BCL-XL selective inhibitor A-1331852}

The C666-1 cells expressed undetectable levels of BFL-1 (Fig. 1b). Hence, to access the role of BFL-1 in cell survival, the gene was deleted in the HK1 cells. The HK-1 cells were transfected with two independent single-guide RNAs (sgRNAs) targeting different regions of the human BFL-1 gene (hereafter the sgRNAs will be referred to as $\mathrm{sg} B F L-1 \# 1$ and $\mathrm{sg} B F L-1 \# 2)$. BFL-1 mutagenesis was verified by conventional Sanger DNA sequencing and gene expression of $B F L-1$ in the parental and $B F L-1$ deleted cells were verified by qPCR. The sgBFL-1\#1 and sgBFL-1\#2 resulted in $\sim 98 \%$ and $80 \%$ reduction in $B F L-1$ expression, respectively (Fig. 5a).

The sgBFL-1\#1 and sgBFL-1\#2 cells were treated with increasing concentrations of either ABT-199 or A1331852 to access whether BFL-1 deletion sensitized NPC cells to these inhibitors. The HK-1 parental cells were resistant to single agent treatment of ABT-199 and A-1331852 (Fig. 5b-c). The sgBFL-1\#1 (Fig. 5b \& Table S4) and the sgBFL-1\#2 (Fig. 5c \& Table S4) cells were weakly sensitized to ABT-199. However, the sgBFL-1\#1 (Fig. 5b \& Table S4) and the sgBFL-1\#2 (Fig. 5c \& Table S4) cells were sensitized to A1331852 by $\sim 5$-fold and 4-fold, respectively indicating that the BFL-1 and BCL-XL may be important for NPC cell survival.

\section{Discussion}

Given that different cell population is addicted to different anti-apoptotic protein(s) for survival, it is crucial to determine the anti-apoptotic proteins that NPC cells depend for survival. We combined the CRISPR/Cas9 technique and BH3 mimetics to delineate the individual contributions of the anti-apoptotic proteins for NPC survival. BH3 mimetics, given their highly selective inhibition of the anti-apoptotic proteins, provide a chemical toolkit to parse the individual contributions of the anti-apoptotic proteins for cancer cell survival.

In our hands, complete deletion of $M C L-1$ alone did not kill the HK-1 sgMCL-1\#2 cells, implying that other anti-apoptotic proteins may be compensating for the loss of MCL-1. Similarly, the NPC parental cell lines were insensitive to single agent treatment of S63845. Moreover, the NPC parental cell lines were also insensitive to single agent treatment of ABT-199 and A-1331852. Collectively, these findings reveal that NPC cells depend on more than one anti-apoptotic protein for survival. The HK-1 sgMCL-1\#2 cells were weakly sensitized to ABT-199 but were sensitized to A-1331852 indicating that MCL-1 and BCL-XL may be important for NPC cell survival. The findings obtained with the MCL-1 manipulated cell line was 
analogous to the findings obtained with the parental NPC cell lines. The effect of co-inhibition MCL-1 and BCL-XL with S63845 and A-1331852 were more profound as both the HK-1 and C666-1 cell lines were instantly killed at nanomolar drug combination concentrations. Both the HK-1 and C666-1 cells also responded to co-inhibition of MCL-1 and BCL-2 but synergism was mostly attained at higher concentrations of the drugs $(>1 \mu \mathrm{M})$ demonstrating that inhibition of MCL-1 and BCL-XL are better therapeutic targets for killing NPC cells.

Given the promising sensitivity of the NPC cells to co-inhibition of BCL-XL and MCL-1 in monolayer culture, co-inhibition of BCL-XL and MCL-1 was tested in NPC spheroids. The sensitization obtained in the monolayer culture were similar with findings obtained with the spheroid studies. Spheroids generated from the HK-1 cells were sensitized to A-1331852 by S63845 and vice versa, indicating that the combination may be effective in vivo. Collectively, our data demonstrated that there was a greater response of the NPC cell lines to co-inhibition of MCL-1 and BCL-XL. Similar to our findings, a number of recent studies demonstrated that solid tumours depend on BCL-XL and MCL-1 for survival and pharmacological inhibition of these proteins resulted in cell killing. For example, co-targeting MCL- 1 and $B C L-X L$ resulted in reduced cell viability of cells isolated from squamous cell carcinoma of the head and neck (SCCHN), in in vitro and in vivo models [20]. Combination targeting MCL-1 and BCL-XL synergistically killed melanoma cell lines in vitro [21]. Paediatric solid tumour cell lines, namely rhabdomyosarcoma, Ewing sarcoma, osteosarcoma and neuroblastoma cell lines were co-dependant on BCL-XL and MCL-1 for survival. Co-treatment with A-1331852 and S63845 induced rapid cell killing of these cell lines in vitro and in vivo models [22]. Our own study demonstrated that co-inhibition of MCL-1 and BCL-XL was crucial for killing cervical cancer cell lines [16,17].

One possible mechanism that may have led to the profound loss of cell viability of the NPC cells, as a consequence of co-inhibition of MCL-1 and BCL-XL, could be explained by the relationship between BIM, MCL-1 and BCL-XL. MCL-1 and BCL-XL sequester BIM and inhibition of either MCL-1 or BCL-XL, liberates $\mathrm{BIM}$ which can be later sequestered by the uninhibited protein [23-25]. We speculate that co-targeting MCL-1 and BCL-XL in the NPC cell lines may have led to complete freedom of BIM which in turn led to activation of BAX and BAK which resulted in the activation of cell death in the NPC cells.

Although majority of solid tumours depend on BCL-XL and MCL-1 for survival, targeting BCL-XL and MCL1 in the clinic may be cumbersome for multiple reasons. MCL-1 and BCL-XL are crucial for the survival of not only tumour cells, but also for non-malignant cells. Hence, targeting these molecules are toxic to normal cells. For example, inhibition of BCL-XL results in thrombocytopenia $[26,27]$ and inhibition of MCL-1 was reported to result in cardiac toxicity $[28,29]$. Co-inhibition of BCL-XL and MCL-1 was previously reported to result in fatal hepatotoxicity [30]. There are a few strategies to overcome this issue. Our findings show that cell killing can be achieved at very low doses (nanomolar ranges) of S63845 and A-1331852, which may not be sufficient to cause thrombocytopenia or cardiac toxicity. A recent finding demonstrated that co-inhibition of MCL-1 and BCL-XL was feasible in zebrafish model of head and neck squamous cell carcinoma [20] and embryonic chicken model of rhabdomyosarcoma (RMS) [22]. Another strategy would be to co-inhibit BCL-2 and MCL-1. Although the effect of inhibiting BCL-2 and MCL-1 was 
not as pronounced as inhibiting BCL-XL and MCL-1, combination of ABT-199 and S63845 synergistically inhibited cell proliferation of the NPC cell lines. Hence, targeting BCL-2 and MCL-1 could be an alternative treatment strategy. Although still preliminary, co-targeting BCL-XL and BFL-1 could be an alternate strategy to reduce toxicities associated with co-inhibiting MCL-1 and BCL-XL. Targeting BFL-1 in addition to $B C L-X L$ should not add significant toxicity to cells, as loss of $B F L-1$ was reported to only reduce certain T-cell subpopulations and dendritic cells [31]. BFL-1 by large is not essential for the development and survival of most normal and healthy tissues [31] compared to MCL-1 and BCL-XL which are crucial for survival of some non-malignant cells. Hence, targeting BFL-1 and BCL-XL may result in acceptable therapeutic window without causing much damage to the normal cells.

There was no notable reduction in the cell viability observed in the BFL-1 manipulated cells despite the complete decrease in BFL-1 expression level, especially in the HK-1 sgBFL-1\#1 cells. The sgBFL-1\#1 cells were equally viable as the $s g B F L-1 \# 2$ cells which did not display complete $B F L-1$ reduction, suggesting that the BFL-1 alone is not essential for NPC cell survival, highlighting the need for additional inhibition of other anti-apoptotic proteins in this context. Treatment with A-1331852 and not ABT-199, sensitized both sgBFL-1\#1 and sgBFL-1\#2 cells to the drug, indicating that BFL-1 and BCL-XL may be necessary for NPC cell survival. The role of BFL-1 in cancer cell survival is not consistent when different cell populations are considered. For example, RNAi-mediated silencing of BFL-1 was not sufficient to induce cell death in lymphoma cell lines. However, pharmacological inhibition of MCL-1 with AZD5991, in combination with dose-dependent BFL-1 knockdown, induced cell killing, demonstrating that BFL-1 and MCL-1 were crucial for the survival of the lymphoma cells. The same study reported that the BFL-1 knockdown in lymphoma cells were insensitive to pharmacological inhibition of BCL-XL and BCL-2 [32]. In a separate study, CRISPR/Cas9 mediated knockout of $B F L-1$ in melanoma cells were not sensitized to pharmacological inhibition of MCL-1, BCL-XL and BCL-2 indicating that the melanoma cells do not rely on BFL-1 for survival [21].

The cruciality of BCL-XL and BFL-1 for NPC cell survival still requires further interrogation. The contribution of BFL-1 for NPC cell survival was only interrogated in one NPC cell line, hence our findings may be a cell-type dependant effect. The functional importance of BFL-1 should be interrogated in additional NPC cell lines to omit the possibility of cell-type dependant effect. Furthermore, inhibition of $B F L-1$ might not be phenotypically severe compared to the immediate inhibition with a BH3-mimetic as CRISPR-modified cell lines are subjected to evolutionary pressure over the course of days or weeks. The intense pressure of Cas9-induced modifications may select for secondary mutations that blunt any antiproliferative consequences of the original mutation [33]. Collectively, these limitations restrict the ability to fully comprehend the contribution of $B F L-1$ for NPC cell survival. As aforementioned, pharmacological inhibition of a target protein induces a more severe phenotype compared to CRISPR/Cas9 manipulation. However, the unavailability of BFL-1 selective $\mathrm{BH} 3$-mimetic halts any further investigation on the role of BFL-1 in promoting survival of NPC cells.

Taken together, our findings reiterate the importance of determining the anti-apoptotic proteins that cancer cells depend for survival so that the cells can be targeted optimally to achieve maximal cell killing. 
In the context of NPC, MCL-1 and BCL-XL are crucial for NPC cell survival and co-targeting these proteins kill cells at low drug doses. Although preliminary, interrogation on the role of $B F L-1$ for NPC cell survival, provided a new insight on the potential of BFL-1 as a therapeutic target for NPC treatment.

\section{Declarations}

Acknowledgements: We would like to thank Professor Dr. George Sai Wah Tsao (University of Hong Kong, Pokfulam, Hong Kong, China) for providing the NPC cell lines HK-1.

Authors' contributions: SFAR: performed the experiments, data analysis, writing - original draft; AA: helped SFAR with experiments which involved the CRISPR/Cas9 technique; K-WL: resources; GA: resources; NM-K: conceptualization, data analysis, resources, writing - original draft, funding acquisition.

Funding: This study was funded by the Fundamental Research Grant Scheme (FRGS), Ministry of Education, Malaysia (Grant number: 203/PBIOLOGI/601228), Universiti Sains Malaysia Research University (RU) grant (Grant number: 1001/PBIOLOGI/8012268) and L'oréal-UNESCO National Fellowship for Women in Science Malaysia (304/PBIOLOGI/650853/L117).

Data availability: All data are available from the corresponding author upon reasonable request.

Consent for publication: Not applicable

Competing interests: The authors declare no conflict of interest.

\section{References}

1. Global Cancer Observatory: Cancer Today. Lyon, France: International Agency for Research on Cancer (IARC). Available from https://gco.iarc.fr/today, accessed [02 November 2021].

2. Al-Sarraf M, LeBlanc M, Giri PG et al. Chemoradiotherapy versus radiotherapy in patients with advanced nasopharyngeal cancer: phase III randomized Intergroup study 0099. Journal of Clinical Oncology. 1998; https://doi.org/10.1200/jco.1998.16.4.1310.

3. Kurschat C, Metz A, Kirschnek S et al. Importance of Bcl-2-family proteins in murine hematopoietic progenitor and early B cells. Cell Death \& Disease. 2021; https://doi.org/10.1038/s41419-021-040798

4. Sheu LF, Chen A, Meng CL et al. Analysis of bcl-2 expression in normal, inflamed, dysplastic nasopharyngeal epithelia, and nasopharyngeal carcinoma: association with p53 expression. Human Pathology. 1997; https://doi.org/10.1016/s0046-8177(97)90078-2.

5. Yu Y, Dong W, Li X et al. Significance of c-Myc and Bcl-2 protein expression in nasopharyngeal carcinoma. Archives of Otolaryngology-Head \& Neck Surgery. 2003; https://doi.org/10.1001/archotol.129.12.1322 
6. Fan SQ, Ma J, Zhou J et al. Differential expression of Epstein-Barr virus-encoded RNA and several tumor-related genes in various types of nasopharyngeal epithelial lesions and nasopharyngeal carcinoma using tissue microarray analysis. Human Pathology. 2006; https://doi.org/10.1016/j.humpath.2006.01.010.

7. Chen MK, Yang SF, Lai JC et al. Expression of bcl-2 correlates with poor prognosis and modulates migration of nasopharyngeal carcinoma cells. Clinica Chimica Acta. 2010; https://doi.org/10.1016/j.cca.2009.12.010.

8. Hu ZY, Zhu XF, Zhong ZD et al. ApoG2, a novel inhibitor of antiapoptotic Bcl-2 family proteins, induces apoptosis and suppresses tumour growth in nasopharyngeal carcinoma xenografts. International Journal of Cancer. 2008; https://doi.org/10.1002/ijc.23752.

9. Yip KW, Mocanu JD, Au PB et al. Combination bcl-2 antisense and radiation therapy for nasopharyngeal cancer. Clinical Cancer Research. 2005; https://doi.org/10.1158/1078-0432.CCR-051266.

10. Xiang BLS, Lo K-W, Khoo AB-S et al. Single Agent and Synergistic Activity of Maritoclax with ABT-263 in Nasopharyngeal Carcinoma (NPC) Cell Lines. Tropical Life Sciences Research. 2020; https://doi.org/ 10.21315/tlsr2020.31.3.1.

11. Wang $Y$, Wang Y, Fan $X$ et al. ABT-199-mediated inhibition of Bcl-2 as a potential therapeutic strategy for nasopharyngeal carcinoma. Biochem Biophys Res Commun. 2018; https://doi.org/10.1016/j.bbrc.2018.07.027.

12. Montero $\mathrm{J}$ and Letai $\mathrm{A}$. Why do BCL-2 inhibitors work and where should we use them in the clinic? Cell Death Differ. 2018; https://doi.org/10.1038/cdd.2017.183.

13. Aubrey BJ, Kelly GL, Kueh AJ et al. An inducible lentiviral guide RNA platform enables the identification of tumor-essential genes and tumor-promoting mutations in vivo. Cell Rep. 2015; https://doi.org/10.1016/j.celrep.2015.02.002.

14. Leverson JD, Phillips DC, Mitten MJ et al. Exploiting selective BCL-2 family inhibitors to dissect cell survival dependencies and define improved strategies for cancer therapy. Sci. Transl. Med. 2015; https://doi.org/10.1126/scitransImed.aaa4642.

15. Daker M, Ahmad M and Khoo AS. Quercetin-induced inhibition and synergistic activity with cisplatin - a chemotherapeutic strategy for nasopharyngeal carcinoma cells. Cancer Cell International. 2012; https://doi.org/10.1186/1475-2867-12-34.

16. Lian BSX, Yek AEH, Shuvas $H$ et al. Synergistic anti-proliferative effects of combination of ABT-263 and MCL-1 selective inhibitor A-1210477 on cervical cancer cell lines. BMC Res Notes. 2018; https://doi.org/10.1186/s13104-018-3302-0.

17. Abdul Rahman SF, Muniandy K, Soo YK et al. Co-inhibition of BCL-XL and MCL-1 with selective BCL-2 family inhibitors enhances cytotoxicity of cervical cancer cell lines. Biochem Biophys Rep. 2020; https://doi.org/10.1016/j.bbrep.2020.100756.

18. Lucas KM, Mohana-Kumaran N, Lau D et al. Modulation of NOXA and MCL-1 as a strategy for sensitizing melanoma cells to the BH3-mimetic ABT-737. Clinical Cancer Research. 2012; 
https://doi.org/10.1158/1078-0432.CCR-11-1166

19. McCloy RA, Rogers S, Caldon CE et al. Partial inhibition of Cdk1 in $\mathrm{G} 2$ phase overrides the SAC and decouples mitotic events. Cell Cycle. 2014; https://doi.org/10.4161/cc.28401.

20. Carter BZ, Mak PY, Tao W et al. Targeting MCL-1 dysregulates cell metabolism and leukemia-stroma interactions and resensitizes acute myeloid leukemia to BCL-2 inhibition. Haematologica. 2020; https://doi.org/10.3324/haematol.2020.260331.

21. Lee EF, Harris TJ, Tran S et al. BCL-XL and MCL-1 are the key BCL-2 family proteins in melanoma cell survival. Cell death \& disease. 2019; https://doi.org/10.1038/s41419-019-1568-3.

22. Kehr S, Haydn T, Bierbrauer A et al. Targeting BCL-2 proteins in pediatric cancer: Dual inhibition of BCL-XL and MCL-1 leads to rapid induction of intrinsic apoptosis. Cancer letters. 2020; https://doi.org/10.1016/j.canlet.2020.02.041.

23. Jeng PS, Inoue-Yamauchi A, Hsieh JJ et al. BH3-dependent and independent activation of BAX and BAK in mitochondrial apoptosis. Current opinion in physiology. 2018; https://doi.org/10.1016/j.cophys.2018.03.005.

24. Inoue-Yamauchi A, Jeng PS, Kim K et al. Targeting the differential addiction to anti-apoptotic BCL-2 family for cancer therapy. Nature communications. 2017; https://doi.org/10.1038/ncomms16078.

25. Iyer S, Uren RT, Dengler MA et al. Robust autoactivation for apoptosis by BAK but not BAX highlights BAK as an important therapeutic target. Cell death \& disease. 2020; https://doi.org/10.1038/s41419020-2463-7.

26. Zhang $\mathrm{H}$, Nimmer PM, Tahir SK et al. Bcl-2 family proteins are essential for platelet survival. Cell Death \& Differentiation. 2007; https://doi.org/10.1038/sj.cdd.4402081.

27. Mason KD, Carpinelli MR, Fletcher JI et al. Programmed anuclear cell death delimits platelet life span. Cell. 2007; https://doi.org/10.1016/j.cell.2007.01.037.

28. Thomas RL, Roberts DJ, Kubli DA et al. Loss of MCL-1 leads to impaired autophagy and rapid development of heart failure. Genes \& development. 2013; https://doi.org/10.1101/gad.215871.113.

29. Wang $X$, Bathina M, Lynch J et al. Deletion of MCL-1 causes lethal cardiac failure and mitochondrial dysfunction. Genes \& development. 2013; https://doi.org/10.1101/gad.215855.113.

30. Weeden CE, Ah-Cann C, Holik AZ et al. Dual inhibition of BCL-XL and MCL-1 is required to induce tumour regression in lung squamous cell carcinomas sensitive to FGFR inhibition. Oncogene. 2018; https://doi.org/10.1038/s41388-018-0268-2.

31. Schenk RL, Tuzlak S, Carrington EM et al. Characterisation of mice lacking all functional isoforms of the pro-survival BCL-2 family member A1 reveals minor defects in the haematopoietic compartment. Cell Death Differ. 2017; https://doi.org/10.1038/cdd.2016.156.

32. Boiko S, Proia T, San Martin M et al. Targeting Bff-1 via acute CDK9 inhibition overcomes intrinsic BH3-mimetic resistance in lymphomas. Blood. 2021; https://doi.org/10.1182/blood.2020008528.

\section{Figures}




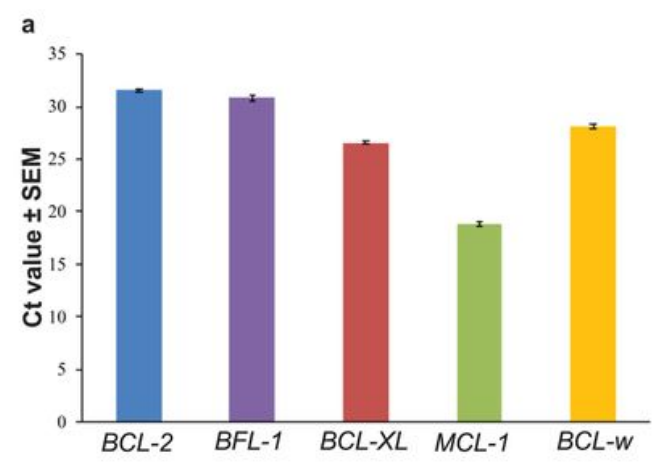

d
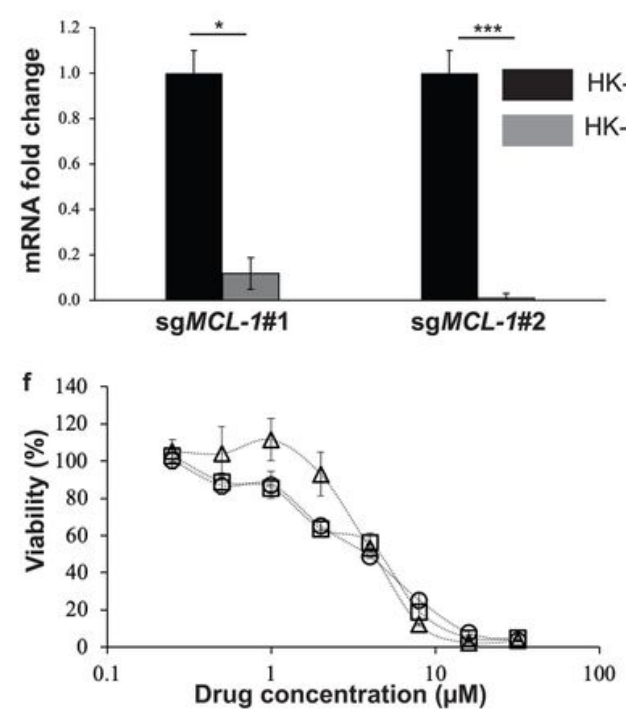

b

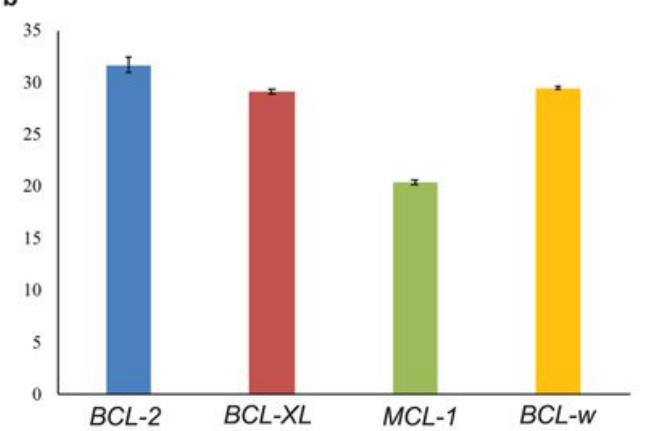

e c

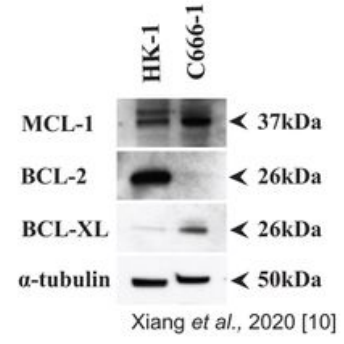

ABT-199

O $s 9 M C L-1 \# 2+A B T-199$

$\mathrm{OA}-1331852$

O $s M C L-1 \# 2$ + A-1331852 g

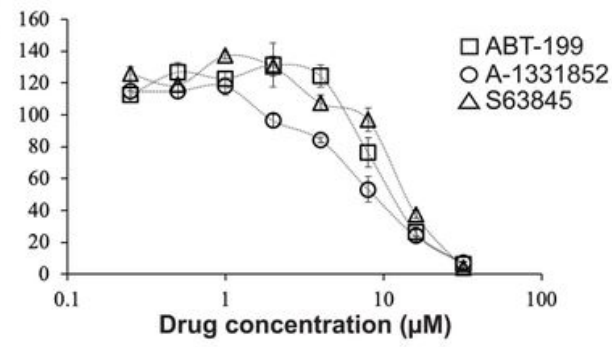

\section{Figure 1}

HK-1 MCL-1 deleted cells were sensitive to treatment of BCL-XL selective inhibitor A-1331852. Expression of the anti-apoptotic genes were determined using the human apoptosis real time PCR array (RT2 Profiler PCR Array). Ct values are inversely proportional to the amount of target gene present in the cells. The (a) HK-1 expressed all of the anti-apoptotic genes and (b) C666-1 expressed all of the anti-apoptotic genes, except BFL-1. Both cell lines expressed high levels of MCL-1. Error bars show standard error of the mean (SEM). (c) Basal expression levels of the anti-apoptotic protein in the NPC cell lines. The basal expression levels of MCL-1, BCL-2 and BCL-XL in the C666-1 and the HK-1 cells were determined by SDS-PAGE gel electrophoresis (this image was taken from [10] with permission). (d) qPCR validation MCL-1 gene deletion in the HK-1 cells. MCL-1 expression levels were normalized to parental cells. Bars indicate mean SEM of three independent experiments. Statistically significant differences in MCL-1 expression between the parental cell line and the MCL-1 knockout cells are shown as ${ }^{\star *} \mathrm{p} \leq 0.001$ or ${ }^{*} \mathrm{p}<0.05$ determined by two-tailed paired T-test. (e) The sensitivity of the HK-1 parental cell line and the HK-1 sgMCL-1\#2 cells were tested to tested to single agent activity of either ABT-199 or A-1331852 (0-32 $\mu \mathrm{M})$. Points represent 
mean SEM of four experiments. (f) HK-1 and (g) C666-1 were treated with increasing concentration of either ABT-199, A-1331852 or S63845 (0-32 $\mu \mathrm{M})$. Points represent mean SEM of four experiments.
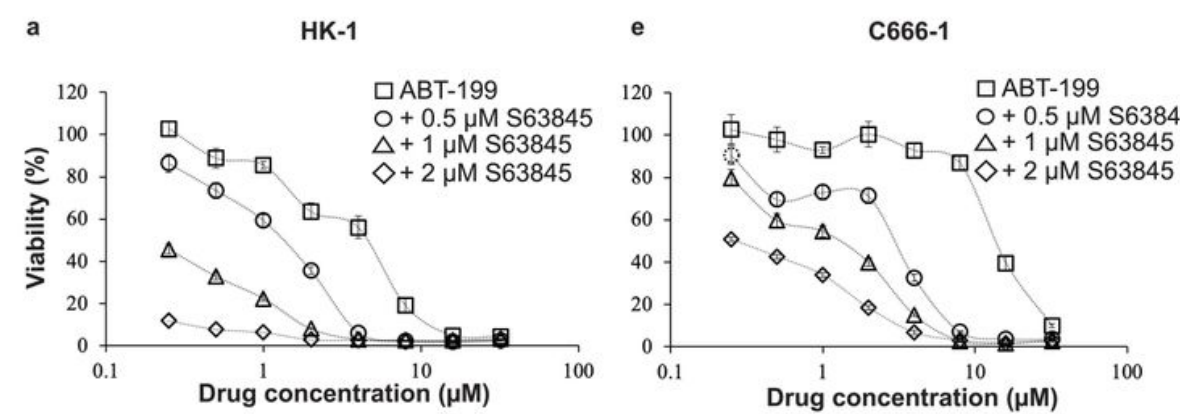

i HK-1

b

f
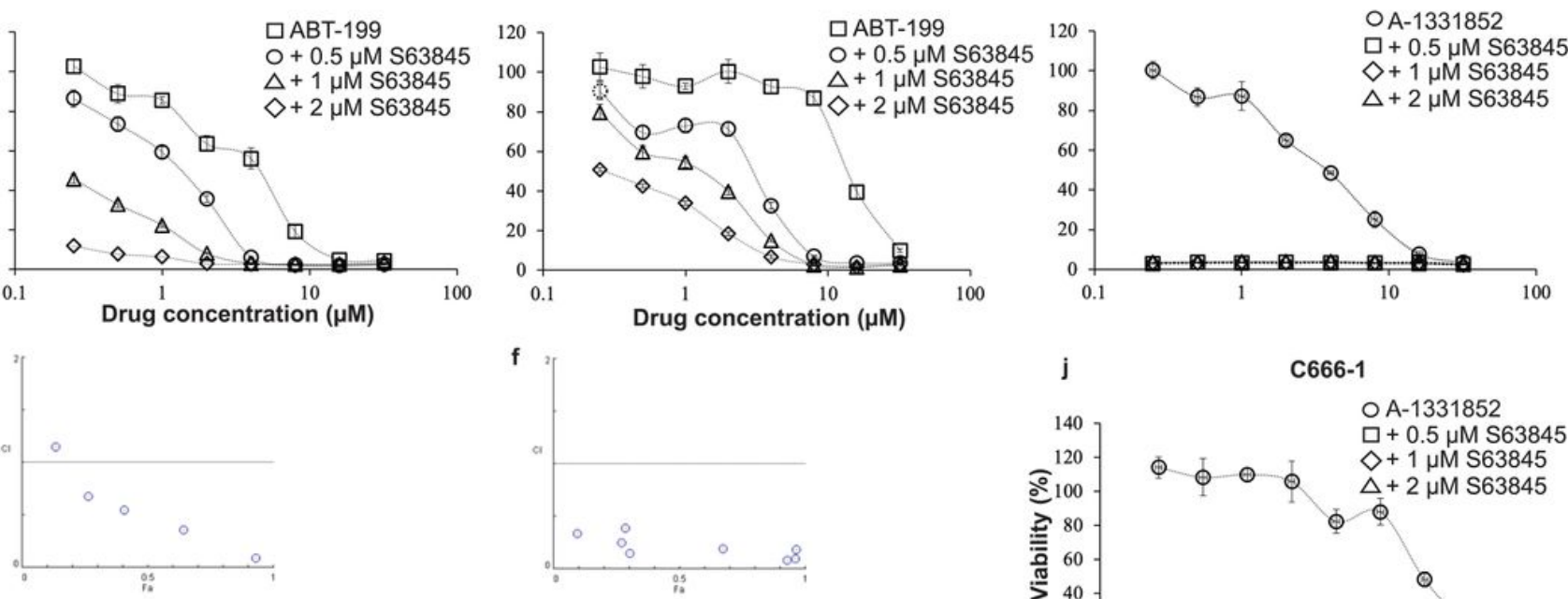

c

g
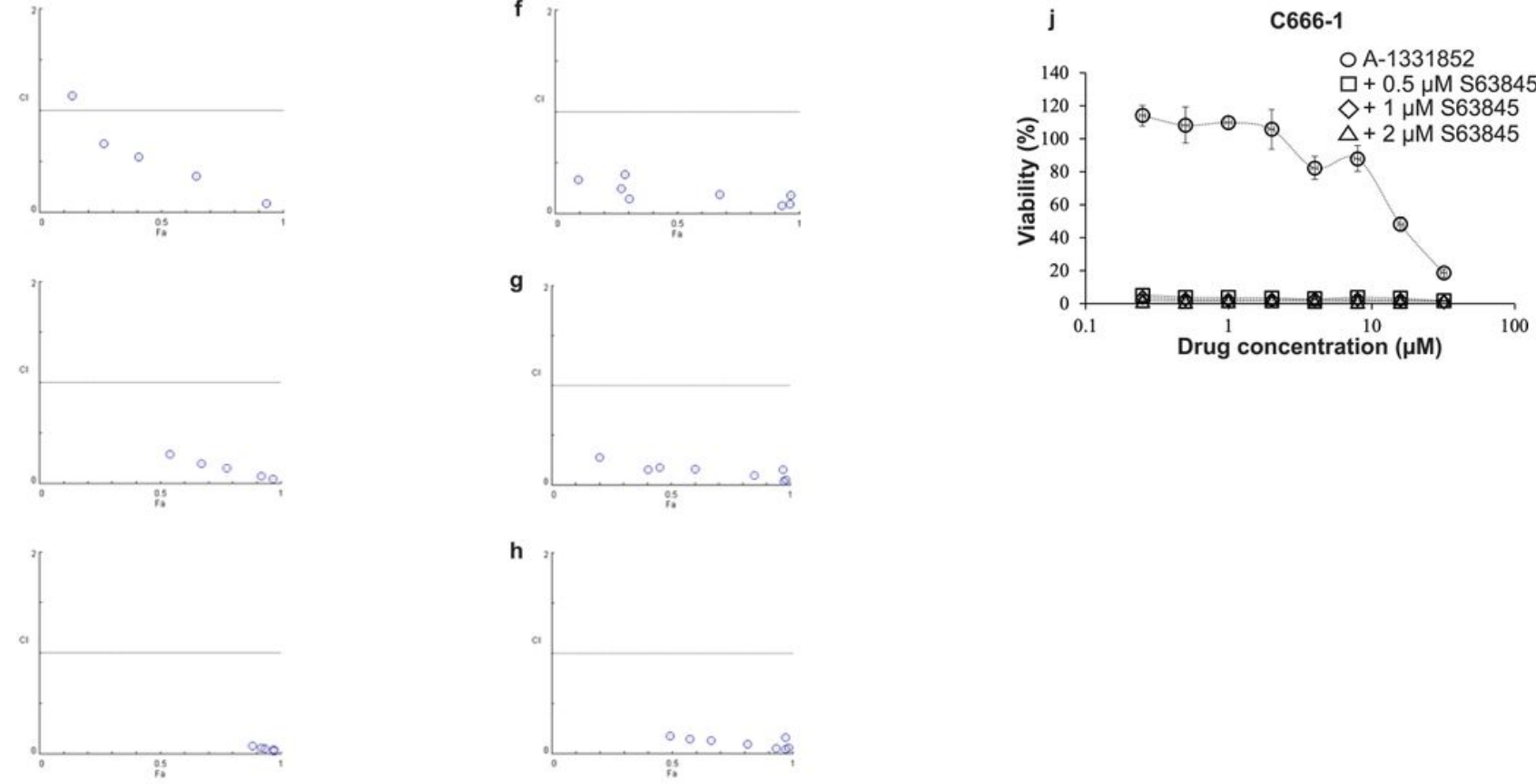

\section{Figure 2}

Co-inhibition of BCL-2 and MCL-1 using ABT-199 and S63845. (a) HK-1 cells were treated with increasing concentrations of ABT-199 $(0-32 \mu \mathrm{M})$ in the presence and absence of S63845. Points represent mean SEM of four experiments. The Fa-Cl plot for sensitization of the HK-1 cells to ABT-199 by either (b) 0.5 $\mu \mathrm{M}$, (c) $1 \mu \mathrm{M}$ or (d) $2 \mu \mathrm{M}$ S63845. The Fa-Cl plots demonstrate the $\mathrm{Cl}$ versus the fraction of cell affected by the combination. The circles represent a specific combination of doses. Data points located below $\mathrm{Cl}=1$ (dotted line) indicate synergism. (e) C666-1 cells were treated with increasing concentrations of ABT$199(0-32 \mu \mathrm{M})$ in the presence and absence of S63845. Points represent mean SEM of four experiments. The Fa-Cl plot for sensitization of the C666-1 cells to ABT-199 by either (f) $0.5 \mu \mathrm{M},(\mathrm{g}) 1 \mu \mathrm{M}$ or (h) $2 \mu \mathrm{M}$ $\mathrm{S} 63845$. The $\mathrm{Fa}-\mathrm{Cl}$ plots demonstrate the $\mathrm{Cl}$ versus the fraction of cell affected by the combination. The circles represent a specific combination of doses. Data points located below $\mathrm{Cl}=1$ (dotted line) indicate synergism. Co-inhibition of BCL-XL and MCL-1 using A-1331852 and S63845. (i) HK-1 and (j) C666-1 cells were treated with increasing concentrations of $A-1331852(0-32 \mu \mathrm{M})$ in the presence and absence of S63845. Points represent mean SEM of four experiments. 
a

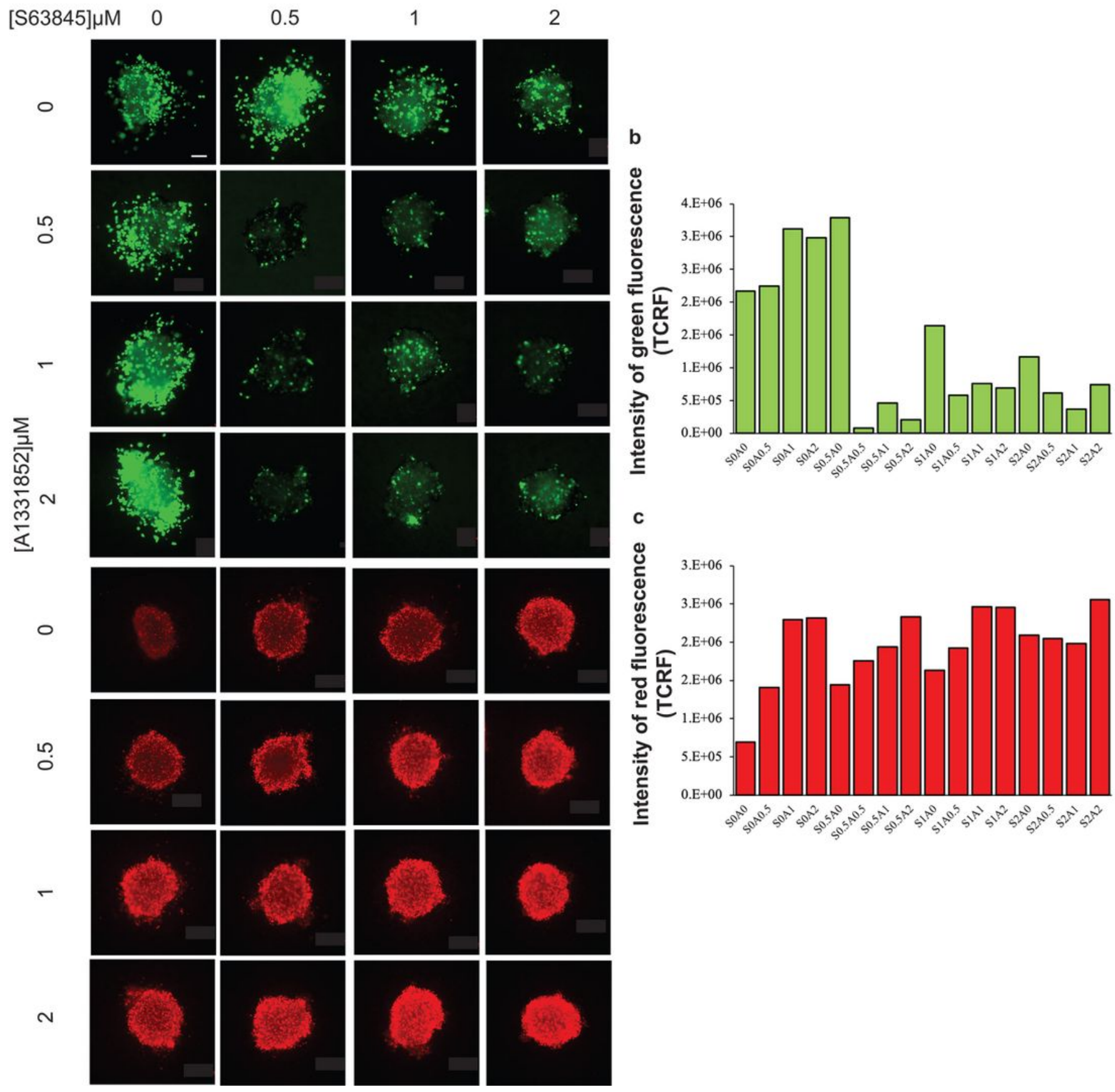

\section{Figure 3}

The effect of combination of S63845 and A-1331852 on the growth and invasion of HK-1 spheroids over three days. (a) The spheroids were treated with single agents $\mathrm{S} 63845$ and A-1331852 and combination of both over three days at the indicated concentrations, $n=2-3$ spheroids per combination. Cell viability was determined using the live/dead assay (Viable cells: stained green by Calcein-AM; Dead cells: stained red by Ethidium-homodimer I). Size bar: $200 \mu \mathrm{m}$. The intensity of (b) green and (c) red fluorescence was measured for each drug combination and presented as TCRF, $n=2$ spheroids per combination. TCRF: Total Corrected Red Fluorescence; " $S$ " denotes S63845 and "A" denotes A-1331852. The numbers next to " $S$ " and " $A$ " indicate the doses of the drug combinations. 


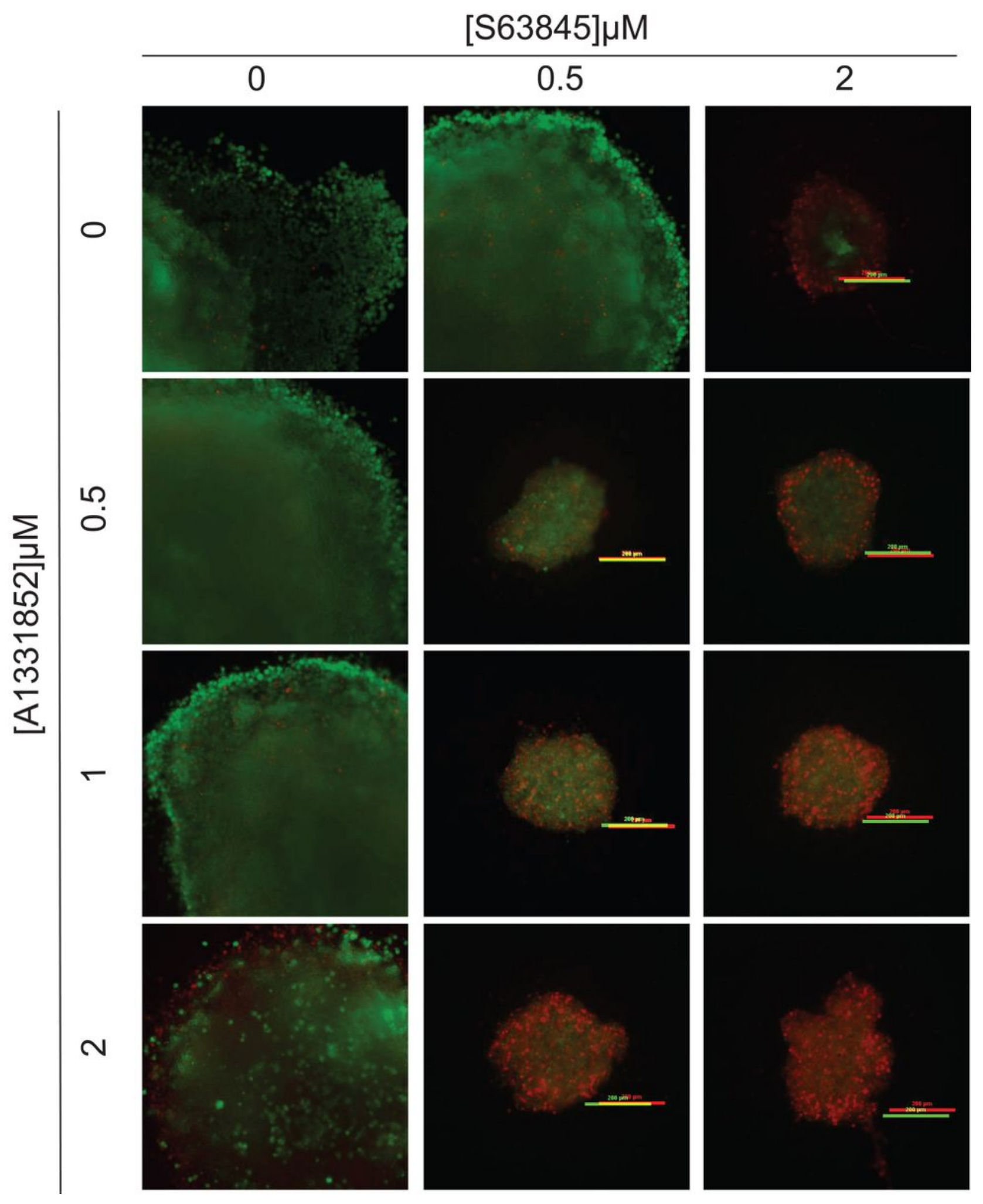

Figure 4

The effect of combination of S63845 and A-1331852 on the growth and invasion of HK-1 spheroids over ten days. Representative spheroid images demonstrating the effect of single agent treatment of A1331852 and $S 63845$ and combinations of both inhibitors over 10 days (merged green and red images). The HK-1 spheroids were treated with single agent of S63845 and A-1331852 and in combination doses 
$(0.5 \mu \mathrm{M}, 1 \mu \mathrm{M} \& 2 \mu \mathrm{M})$. Cell viability was determined using the live/dead assay (Green: calcein-AM, live; Red: ethidium homodimer I, dead) (Size bar $=200 \mu \mathrm{m})$.

a
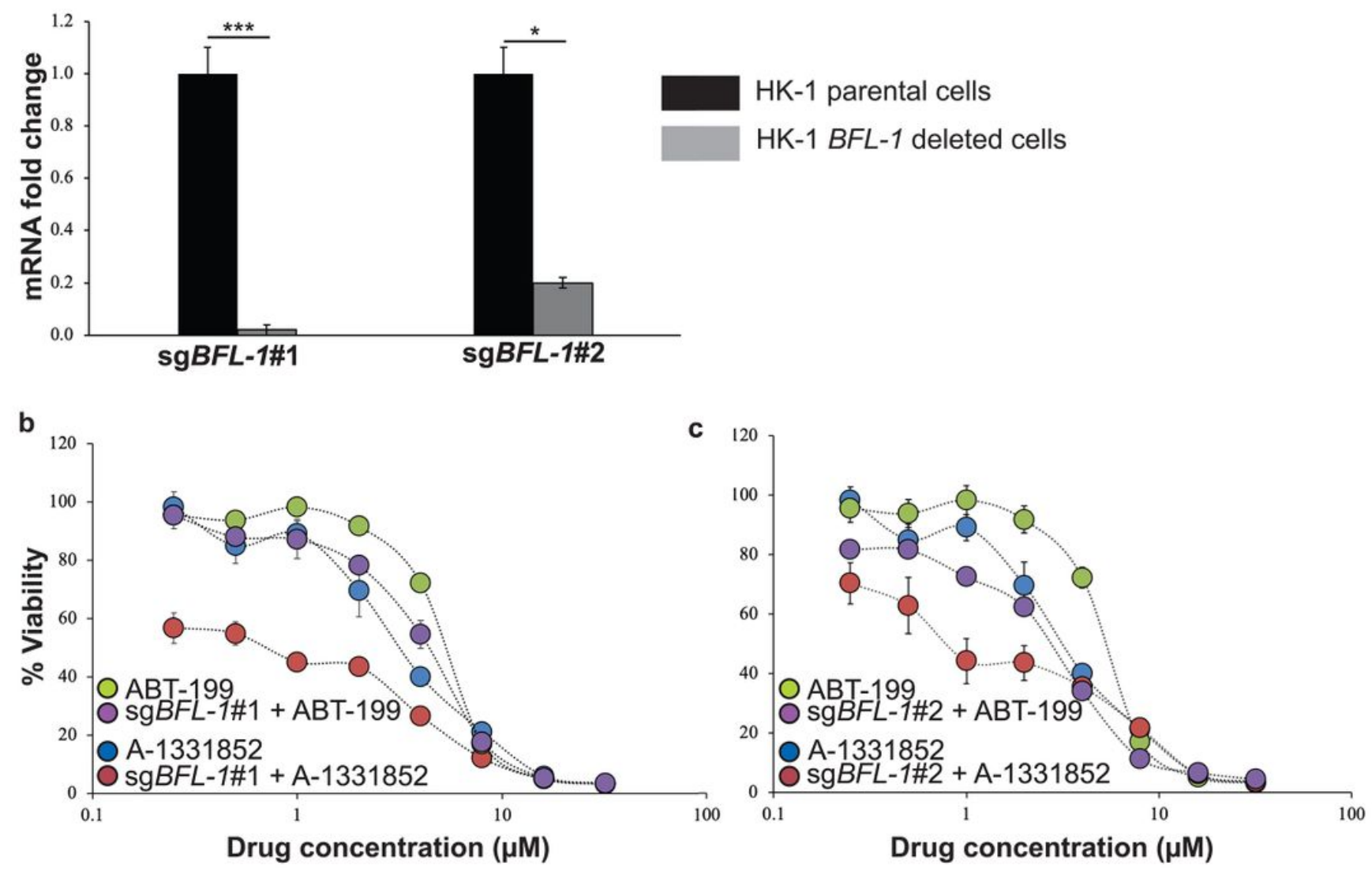

Figure 5

HK-1 BFL-1 deleted cells were sensitive to treatment of BCL-XL selective inhibitor A-1331852. (a) qPCR validation BFL-1 gene deletion in the HK-1 cells. BFL-1 expression levels were normalized to parental cells. Bars indicate mean SEM of three independent experiments. Statistically significant differences in BFL-1 expression between the parental cell line and the BFL-1 knockout cells are shown as ***p $\leq 0.001$ or * $p<0.05$ determined by two-tailed paired T-test. The sensitivity of the HK-1 parental cell line and the (b) HK-1 sgBFL-1\#1 and (c) HK-1 sgBFL-1\#2 cells were tested to tested to single agent activity of either ABT199 or A-1331852 (0-32 $\mu \mathrm{M})$. Points represent mean SEM of four experiments.

\section{Supplementary Files}

This is a list of supplementary files associated with this preprint. Click to download.

- Figs1.eps

- Figs2.eps

- SupplementaryTable1.docx 
- SupplementaryTable2.docx

- SupplementaryTable3.docx

- SupplementaryTable4.docx 